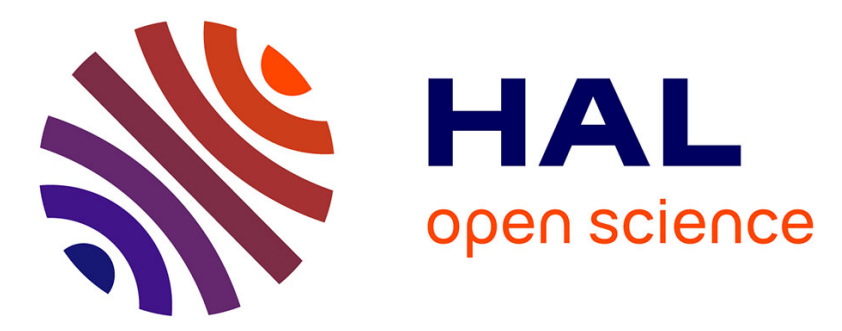

\title{
Application des réseaux de neurones pour l'analyse de l'articulation musicale
}

\author{
B. Kostek
}

\section{To cite this version:}

B. Kostek. Application des réseaux de neurones pour l'analyse de l'articulation musicale. Journal de Physique IV Proceedings, 1994, 04 (C5), pp.C5-597-C5-600. 10.1051/jp4:19945127 . jpa-00252803

\section{HAL Id: jpa-00252803 https://hal.science/jpa-00252803}

Submitted on 1 Jan 1994

HAL is a multi-disciplinary open access archive for the deposit and dissemination of scientific research documents, whether they are published or not. The documents may come from teaching and research institutions in France or abroad, or from public or private research centers.
L'archive ouverte pluridisciplinaire HAL, est destinée au dépôt et à la diffusion de documents scientifiques de niveau recherche, publiés ou non, émanant des établissements d'enseignement et de recherche français ou étrangers, des laboratoires publics ou privés. 


\title{
Application des réseaux de neurones pour l'analyse de l'articulation musicale
}

\section{B. KOSTEK}

Sound Engineering Department, Electronic Faculty, Technical University of Gdansk, Narutowicza 11/12, 80-952 Gdansk, Poland

\begin{abstract}
Knowledge discovery approach was made in the domain of pipe organ sounds. The background of such an approach was reviewed and some experimental results were quoted. Parameterization of pipe organ sounds was discussed, and a feature extraction method was described in terms of parameters derived from both time- and frequency-domain analyses. The knowledge acquisition system principles were described and the neural network algorithms underlying both training and performance processes were presented. The number of necessary data inputs and the structure of neural net were studied in order to obtain the demanded accuracy of results. Experimental results have shown that the technique applied to the analysis of musical events in pipe organ sound might form an approach to the domain of automatic recognition of events in sound.
\end{abstract}

\section{INTRODUCTION}

Le developpement des méthodes appliquées dans l'intelligence artificielle autorise une nouvelle approche de l'analyse des signaux de musique, et en particulier des régimes transitoires. L'étude des régimes transitoires des sons de musique est par sa méthode plus dificile que celle des régimes permanents. Cependant, à cause du fait que les régimes transitoires sont plus interessants du point de vue du jugement subjectif de la qualité et du timbre du son, l'application des techniques du domaine de l'intelligence artificielle parait être justifiée et profitable. En particulier, en exemple d'un outil pour l'estimation quantitative et qualitative des phénomènes non-linéaires qui ont lieu dans les instruments de musique, on peut prendre les programmes qui réalisent les réseaux de neurones.

Les algorithmes de connexion permettent bien en particulier de resoudre les problémes, dont la complexité ne permet pas de réaliser le descriptif exact des phénomènes à l'aide des équations mathématiques. L'exemple d'application de cette technique d'analyse des nonlinéarités peut être l'analyse des régimes transitoires d'attaque dans le son d'orgue. L'objet des études présentées est l'essai d'application des algorithmes de connexion pour détecter le caractère d'une différentiation (dit articulation musicale) dans le jeu d'orgue.

\footnotetext{
${ }^{1}$ Rapport de recherche No. 8 S503 028 06, KBN, Varsovie, Pologne
} 


\section{DETERMINATION DU VECTEUR DES PARAMETRES DU SON D'ORGUE}

Ces dernières années on a vue apparaitre quelques études dans lesquelles on a présenté les analyses des régimes permanents. La méthodologie de ces études provient de l'hypothèse des variation trés lentes du signal de musique en phase de transitoire à l'échelle du temps dans laquelles l'unité du temps est égale à une période de la courbe liée à la composante fondamentale du signal. En conséquence on peut introduire la notion d'un spectre instantanée - à court terme dont les changements donnent l'image objective des changements du timbre du son [1], [2], [3]. La plus ancienne de ces études [1] présente des examples d'évolution des spectres pour un certain nombre des tuyaux sonores d'orgue choisis dans une gamme de quelques composants harmoniques. Le calcul du temps de montée se fait en utilisant une normalisation temporaire liée à une période du fondamental du signal. C'est un procédés profitable car il mène à une independance de l'echelle musicale des sons d'orgue étudiés. Un autre paramétre peut être le caractère qui peut être observé comme un maximum énergetique local du son dont la valeur est supérieure à l'énergie du signal en régime permanent. Les paramètres évoqués ne sont pas liés au spectre du signal, contrairement à la proposition des études de Pollard et de Jansson [3]. Le point de départ sont les résultats de l'analyse de spectre instantanée réalisée plusieurs fois et comprennant toute le transitoire. Le paramétrage consiste à un regroupement des composants harmoniques (1 - fondamental, 2-4, 5n). En résultat on obtient un espace de paramètres de trois dimmensions qui rend l'image de l'évolution des sons pramétrés. Les résultats d'un tel paramètrage peuvent être représentés graphiquement, et donc on peut observer les différences et les ressemblences de deux sons d'orgue.

Dans la litérature on trouve aussi un autre type de paramètrage appliqué pour l'analyse de transitoire [4]. Cela consiste à un certain redressement (dit "lissage") du spectre grâce à l'utilisation des polynômes approximants. De cette façon on peut éviter une définition arbitraire du regroupement des composants harmoniques. Le critère dans ce cas est l'erreur obtenue au procédés d'approximation du spectre moyen d'un range des tuyaux d'orgue. Comme élements du vecteur des paramètres on prend les coéfficients du polynôme, et son évolution peut être suivie sur des courbes à deux dimmensions. Les mèthodes decrites ci-dessus donnent une bonne base de reflexion sur l'approche complète du problème qu'il faut resoudre, est la crèation du vecteur de paramètres comprenant l'information complète au possible au sujet du transitoire analysé du tuyau sonore d'orgue. Il est sans doute profitable d'y mettre les paramètres résultants des relations énergetiques et de spectre, ces dernières sont liées directement au timbre du son. Une telle approche permet aussi de trouver les paramètres liés à la différentiation musicale dans le jeu d'orgue [5], [6].

L'observation des résultats des différentes analyses de transitoire des tuyaux sonores d'orgues mène à la conclusion sur la nécessité de créer un modèle des différentes phases d'évolution temporelle du son. En utilisant le modèle simplifié, la transitoire peut être définie en tant que séquence du signal comprise entre deux états: le silence et le régime permanent; avec cette hypthèse on admet que les variations d'énergie dans le temps sont égales à zero. Cela concerne le cas idéal qui ne prend pas en compte les bruit, distortions, fluctuations, etc, mais montre en même temps le fait que pour le paramètrage des transitoires il est plus facile d'utiliser le modèle de la fonction image de la variation d'énergie dans le temps.

Pour le cas simplifié qui se ramène à une croissance linéaire de l'énergie du son en phase du transitoire il est facile de calculer p.ex. le temps de croissance sans avoir à définir le moment: de début et de fin de cette phase. Dans ce cas on utilise les formules provenant de statistique mathématique, c'est à dire du calcul de la valeur moyenne et de la variance (moments du premier et du sécond ordre). En application au problème discuté il faut utiliser la courbe en fonction du temps de la première dérivé de l'énergie.

Les formules mentionées consiste au calcul des integrales sur l'interval de moins l'infini au plus infini des puissances consecutives de la variable $t$ (temps) multipliée par la fonction de la courbe en fonction du temps de la première dérivé de l'énergie $M_{1}$. Cela donne les résultats suivants: 


$$
M_{1}=\int_{-\infty}^{+\infty} t f(t) d t=\left(b^{2}-a^{2}\right) h / 2
$$

où: $\mathbf{h}$ - croissance de l'énergie par unité de temps,

a - début du transitoire,

$\mathrm{b}$ - fin du transitoire.

Pour les besoins de la normalisation il faut calculer $M_{0}$, ce qui est l'énergie du signal en régime permanent:

$$
M_{0}=\int_{-\infty}^{+\infty} f(t) d t=(b-a) h
$$

Pour calculer le moment $t_{0}$ étant le milieu de la transitoire il faut diviser les deux paramètres par eux-mêmes:

$$
\mathrm{t}_{0}=\mathrm{M}_{1} / \mathrm{M}_{0}=(\mathrm{a}+\mathrm{b}) / 2
$$
formule:

Le temps de croissance nécessite le calcul de deuxième moment central conformement à la

$$
M_{2}=\int_{-\infty}^{+\infty}\left(t-t_{0}\right)^{2} f(t) d t
$$
mène à:

Après les transformation utilisant les formules (1), (2), (3) et (4) on obtient ce qui

$$
t_{\text {croissance }}=b-a=\sqrt{12 M_{2} / M_{0}}
$$

Il parait bon de calculer les moments centraux des ordres superieurs. Par exemple le moment de troisième ordre donne l'image de la symétrie de la courbe analysée et pour le modèle étudie est égal à zero. Il s'avère tout de même qu'un modèle simplifié de cette façon là peut être très rarement appliqué aux transitoires observées en réalité. Il nécessite de rajouter au moins deux phases supplémentaires: disparition partielle et croissance locale de l'énergie juste avant d'atteindre le régime permanent.

\section{PROCEDURE EXPERIMENTALE}

La phase pratique de la réalisation du problème posé consistait en premier lieu à l'extraction du vecteur des paramètres. Pour cela on a enregistré les sons provenant de l'orgue possédant une commande mécanique, en même temps observant de la différentation musicale appliquée. On traite comme suffisant la définition de trois classes liées à la différentation musicale, c'est-à-dire: appuit lent de la touche sur le clavier, moyennement rapide, rapide. Les sons enregistrés ont été stockés numériquement sur un disque dur de l'ordinateur. L'étape suivante de la réalisation était l'utilisation du programme réalisant l'algorithme du réseau de neurones, et ensuite les deux phases suivantes, c'est-à-dire phase 
de l'apprentissage, dit l'entrainement et phase de reconnaisance. La structure du réseau de neurones implementée à la station NeXT est la suivante: entrée lisant le vecteur des paramètres, deux couches cachées et la couche de sortie. Le nombre de neurones dans les couches cachées a été déterminé expérimentalement et est égal à 20 . On présente à l'entrée du réseau le vecteur des paramètres composé de 8 élements, et à la sortie on obtient la réponse sur l'appartennance à une des trois classes de la différentation du transitoire.

Pour vérifier les résultats obténus on a utilisé la statistique $\aleph^{2}$. Un réseau bien enseigné peut supporter la decision de l'homme sur la façon d'articulation dans les expériments sur les nouveaux types de fonctionnement d'orgue, c'est-à-dire la commande en technique fuzzy-logic. L'application de ces deux techniques en même temps assure la possibilité d'informatiser la commande d'orgue en obtenant la qualité du son, proche de celle des instruments d'orgue à fonctionnement mécanique [7].

\section{CONCLUSION}

Les expériences réalisées montrent la possibilité d'appliquer le réseau de neurones à la détection des phénomènes non-linéaires dans le son d'orgue, en particulier, si ces phénomènes ne sont facilement détectables dans une analyse type du signal. On a constaté que le problème principal au niveau de l'application du réseau de neurones est de trouver un vecteur des paramètres caractéristiques du son qui de façon précise decrit les relations énergetiques et de spectre. Les actions ayant pour but l'étude de la relation de la structure du réseau neurones ont permis de constater que l'effort principal devrair être concentré sur la construction de régles de decision. Actuellement on travail sur la vérification expérimentale du vecteur des paramètres et sur l'optimisation des algorithmes de connexion qui servent à la reconnaissance des classes de transitoires. De plus on travail sur l'entrainement du réseau de neurones implémenté. Les résultats obtenus jusqu'à maintenant permettent de dire qu'un réseau entrainé est capable de reconnaitre les transitoires dans le son d'orgue et le qualifie dans une des trois classes de dépendance de la durée du régime transitoire.

\section{BIBLIOGRAPHIE}

1. Keeler J.S., "The Attack Transients of Some Organ Pipes", IEEE Transaction on Audio and Electroacoustics AU-20, No. 5 (1972) 378-391.

2. Pollard H.F. and Jansson E.V., "Analysis and Assessment of Musical Starting Transients ", Acustica 51 (1982) 249-262.

3. Pollard H.F. and Jansson E.V., "A Tristimulus Method for the Specification of Musical Timbre", Acustica 51 (1982) 162-171.

4. Kostek B. and Kaczmarek A., "Listening tests in the computer modelled pipe organ sound", 93rd Audio Eng. Soc. Conv., Preprint 3391, San Francisco 1-4 October 1992, J. Audio Eng. Soc., 40, No. 12 (1992) 1046.

5. Kostek B. and Czyzewski A., "Articulation features in the digitally controlled pipe organ", 90th Audio Eng. Soc. Conv., Preprint 3023, Paris 19-22 February 1991, J. Audio Eng. Soc., 39, No. 5 (1991) 382.

6. Kostek B., "Untersuchungen an Orgeltrakturen unter dem Aspekt musikalischer Artikulierung", DAGA '92, Berlin 16-20 March 1992, Fortschritte der Akustik (1992) 245-248.

7. Kostek B., "Intelligent Control System of the Pipe Organ Instrument", Proc. of the International Workshop on Rough Sets and Knowledge Discovery, RSKD '93, Banff 11-15 October 1993 483-485. 